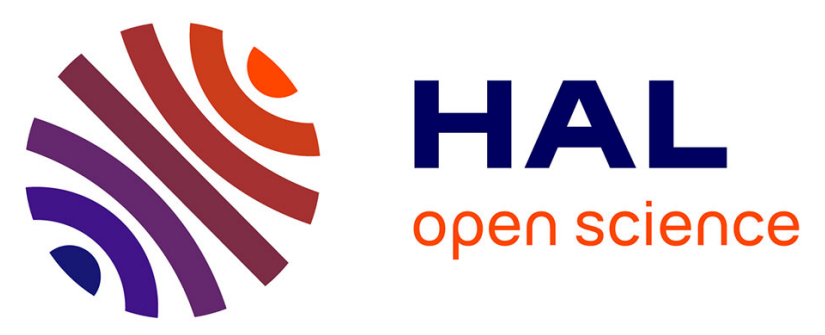

\title{
Measurements, Performance and Analysis of LoRa FABIAN, a real-world implementation of LPWAN
}

Tara Petrić, Mathieu Goessens, Loutfi Nuaymi, Alexander Pelov, Laurent

Toutain

\section{- To cite this version:}

Tara Petrić, Mathieu Goessens, Loutfi Nuaymi, Alexander Pelov, Laurent Toutain. Measurements, Performance and Analysis of LoRa FABIAN, a real-world implementation of LPWAN. PIMRC 2016 : 27th Annual International Symposium on Personal, Indoor, and Mobile Radio Communications, Sep 2016, Valencia, Spain. hal-01331966

\section{HAL Id: hal-01331966 https://hal-imt.archives-ouvertes.fr/hal-01331966}

Submitted on 15 Jun 2016

HAL is a multi-disciplinary open access archive for the deposit and dissemination of scientific research documents, whether they are published or not. The documents may come from teaching and research institutions in France or abroad, or from public or private research centers.
L'archive ouverte pluridisciplinaire $\mathbf{H A L}$, est destinée au dépôt et à la diffusion de documents scientifiques de niveau recherche, publiés ou non, émanant des établissements d'enseignement et de recherche français ou étrangers, des laboratoires publics ou privés. 


\title{
Measurements, Performance and Analysis of LoRa FABIAN, a real-world implementation of LPWAN
}

\author{
Tara Petrić*, Mathieu Goessens*, Loutfi Nuaymi*, Alexander Pelov*, Laurent Toutain* \\ *Telecom Bretagne \\ \{name.surname\}@telecom-bretagne.eu
}

\begin{abstract}
Up to recently, two main approaches were used for connecting the "things" in the growing Internet of Things (IoT) - one based on multi-hop mesh networks, using short-range technologies and unlicensed spectrum, and the other based on long-range cellular network technologies using corresponding licensed frequency bands. New type of connectivity used in LowPower Wide Area networks (LPWAN), challenges these approaches by using low-rate long-range transmission technologies in unlicensed sub-GHz frequency bands. In this paper, we do performance testing on one such star-topology network, based on Semtech's LoRa ${ }^{\text {TM }}$ technology, and deployed in the city of Rennes - LoRa FABIAN. In order to check the quality of service (QoS) that this network can provide, generally and in given conditions, we conducted a set of performance measurements. We performed our tests by generating and then observing the traffic between IoT nodes and LoRa $^{\text {TM }}$ IoT stations using our LoRa FABIAN protocol stack. With our experimental setup, we were able to generate traffic very similar to the one that can be used by real application such as sensor monitoring. This let us extract basic performance metrics, such as packet error rate (PER), but also metrics related specifically to the LoRa physical layer, such as the Received Signal Strength Indicator (RSSI) and Signal to Noise ratio (SNR), within various conditions. Our findings provide insight about the performance of LoRa networks, but also about evaluation methods for these type of networks. We gathered measurement data that we make freely available together with the tools we used.
\end{abstract}

Keywords-IoT communication needs, LPWAN, LoRa performance, long-range radio, access networks.

\section{INTRODUCTION}

Tens of billions of machines and different type of sensors are expected to be deployed and connected in the short term. A large part of these is expected to be covered by Wide Area Networks (WANs). In addition to cellular networks, the recently emerging Low-Power Wide Area Networks (LPWAN) are also a very suitable solution for this type of coverage.

These networks use a physical layer technology that trades bitrate for range, providing a wide coverage (up to tens of kilometers) and energy efficiency at cost of low datarate (in order of hundreds of bits or kbits per second, and, sometimes, much less). LoRa from Semtech
[1] is one prominent technology of this type. LoRa is a Layer 1 Network Protocol designed to work on sub-1GHz spectrum $(109 \mathrm{MHz}, 433 \mathrm{MHz}, 866 \mathrm{MHz}, 915 \mathrm{MHz})$. As those frequencies are globally available, without any licences, they are good candidates for the Internet of Things (IoT) communication needs.

LPWAN's based on LoRa technology, are still insufficiently researched and tested. As far as we are aware, only a few publications are available on this topic.

Introduction and discussion of LoRa LPWAN technology is given by [2]. This work is followed by [3] where a description of an experimental deployment of a LoRa network is provided and a rough estimation of the number of gateways needed to cover a city is done. The performance of LoRa when transmitting through materials such as water and concrete is done in [4]. First short and longrange measurements was conducted by [5], however in a notably open/semi-rural environment. The measurement was done with few or no obstacles concealing the line of sight (LoS).

In this paper we build upon this work, extending it to different areas, conditions, radio parameters and LoRa devices.

We first describe the LoRa FABIAN network setup that was developed and installed by the authors, in cooperation with other colleagues, students and two companies Kerlink 6] and Wi6Labs [7. We give details about the overall architecture, equipment and protocols used. Then, after providing the current radio parameters used in our network, we detailed the measurements done and analyzed the performance observed.

\section{LORA FABIAN}

LoRa FABIAN [8] is a Network Protocol Stack and experimental network setup, deployed in Rennes, France, for IoT needs. Although being mostly designed for LoRa ${ }^{\mathrm{TM}}$ and the associated constraints (most notably, ow bandwidth) it can be reused on top of any Layer 1 technology.

LoRa FABIAN aims at democratizing access to lowpower long-range technologies, by abstracting the network complexity using common Internet protocols such as CoAP, DNS, HTTP(s) etc. IoT objects are designed to run a local CoAP server and client which offers resources and access to the object, which can be used to control the object and interact with its environment. This CoAP server 
is directly accessible from the Internet, using an HTTP translator. That way, it can leverage all web technologies.

\section{A. LoRa FABIAN Architecture}

LoRa FABIAN uses different components that are necessary to test and provide coverage for IoT applications. This include both communicating and gathering data from IoT objects, and connecting them to the Internet (both to send data, and receive remote commands). Therefore, the setup (as shown on Fig. 11) includes: IoT objects, LoRa IoT stations (that can communicate with the objects), and components that enable two-way communication with the Internet and its services (the Gateway and the Service node).

The experimental IoT objects are composed of an Arduino [9] and a FroggyFactory [10 LoRa Shield running a modified version of contiki OS 11]. The LoRa Shield handles the low level communication and low level network access (registration, authorization, etc.), following a mechanism, that was designed by the authors and proposed in [12]. It forwards the data traffic to the Arduino, which allows the user to prototype application in a simple environment, by defining CoAP [13 based REST [14 resources, that can then be accessed from the Internet. In the same way, the user can use the Arduino interface to send data traffic to the network. The LoRa shield can have an embedded antenna or an external, soldered-on, antenna that provides additional gain.

The LoRa IoT station, built by Kerlink [6] is a LoRa ${ }^{\mathrm{TM}}$ antenna, that has the ability to connect with the outside via Ethernet or 3G. It can listen and send traffic (to/from IoT objects) using LoRa ${ }^{\mathrm{TM}}$ technology through multiple frequencies and code-rate at the same time. It redirects this traffic through a simple UDP tunnel to/from the LoRa FABIAN Gateway exporting data and meta-data in a simple JSON 15 text format.

The Gateway can communicate with many LoRa IoT stations in order to concentrate traffic for a global area like a city or a country. It is in charge of sending beacons to inform IoT objects about the network availability and to receive their registration messages, informing IoT objects they may have to change their radio parameters (frequency, code rate). All the traffic intended for the registered IoT object (downstream), and all the packets coming from the objects (upstream) then go through the Gateway. The downstream traffic can be stored here, to allow the IoT objects to be powered off and receive messages once they awake. Once registered, all the messages coming from the IoT objects are redirected using HTTPS tunnels to the Service node. The code for the gateway was written in Java by the authors and it is based on Californium, as well as other libraries (JPA, H2 database,...)

The Service node, where every IoT object's DNS name points to, runs a simple translator from HTTP to CoAP protocol (and vice versa) written in Java. The code was developed by the authors and is based on the Californium [16] proxy. It receives traffic from the Internet and redirects it to the right Gateway that the object is associated to, and vice versa.

Currently, the LoRa FABIAN network (as shown in Fig. 1) consists of three LoRa IoT stations (in different locations), one Gateway, one Service node and number of IoT objects (used mostly for testing purposes).

\section{B. Specification and radio parameters of LoRa IoT stations} and experimental Io $T$ objects

LoRa IoT stations are running a customized Linux System on top of an ARM platform that uses a Semtech SX1276 chip for providing the LoRa connectivity with a $5 \mathrm{dBm}$ antenna.

Out of the three LoRa stations hosted by LoRa FABIAN, two are used for the production network. They are hosted by Télé Diffusion de France (TDF) 17] on two high points (one in 9 avenue Jean Janvier, 35000 Rennes, France, elevation: 85m, and the other in 2 Rue du Clos Courtel, 35510 Cesson-Sévigné, Rennes, France, elevation: $160 \mathrm{~m})$. The last LoRa station is situated on the roof of Télécom Bretagne (2 Rue de la Châtaigneraie, 35510 Cesson-Sévigné. Rennes. France., elevation: 55m) used as an experimental platform.

All those antennas are configured to use the following parameters:

- Bandwidth: 868.1 to $868.225 \mathrm{Mhz}$

- Channel size: $125 \mathrm{kHz}$

- Spreading Factor (SF):

- Sending (downlink), fixed $=7$

- Receiving, variable, from 7 to 12

- Coding Rate: $4 / 7$ (for sending, but can receive $4 / 5$ to $4 / 8)$

- Transmitting Power: $14 \mathrm{dBm}$

The IoT object, for now, in order to receive traffic from LoRa stations, needs to use the same parameters, in terms of frequency, coding rate, channel size and SF. For transmitting they use a fixed power of $14 \mathrm{dBm}$. The FroggyFactory LoRa shield comes with an embedded antenna, however, in our setup, some shields were modified to have an external, soldered-on, antenna to test the performance gain.

\section{Use OF LORA AND COAP FOR SIGNALING AND DATA}

LoRa FABIAN uses two different kind of messages to communicate between nodes, over the LoRa technology.

\section{A. CoAP for signaling}

Gateway and objects are exchanging control messages, using CoAP, like network announces (beacons), nodes registration, nodes control messages (e.g. radio parameter changes).

\section{B. CoAP for data}

As objects also implement a CoAP server and client, they can use it to communicate with the Service node and offer their services directly to the Internet. 


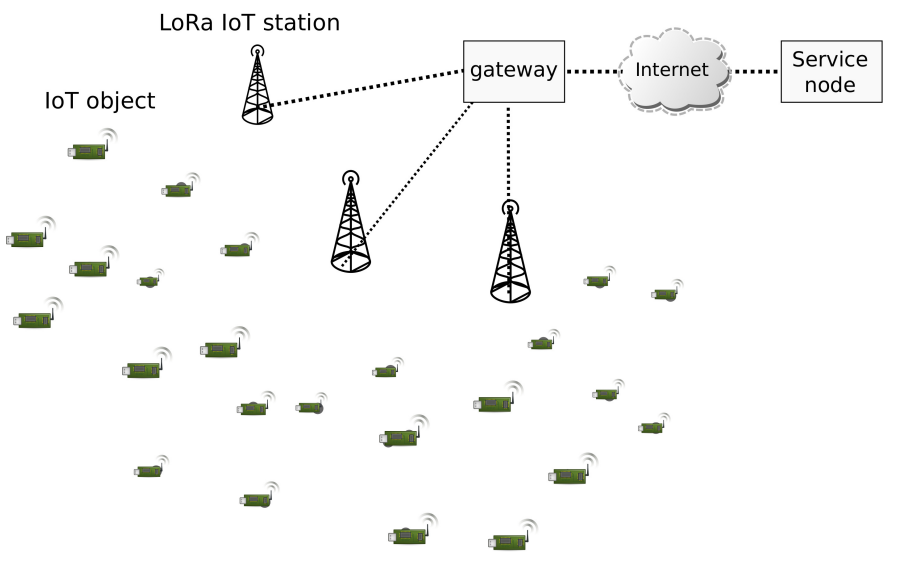

Fig. 1. LoRa FABIAN architecture

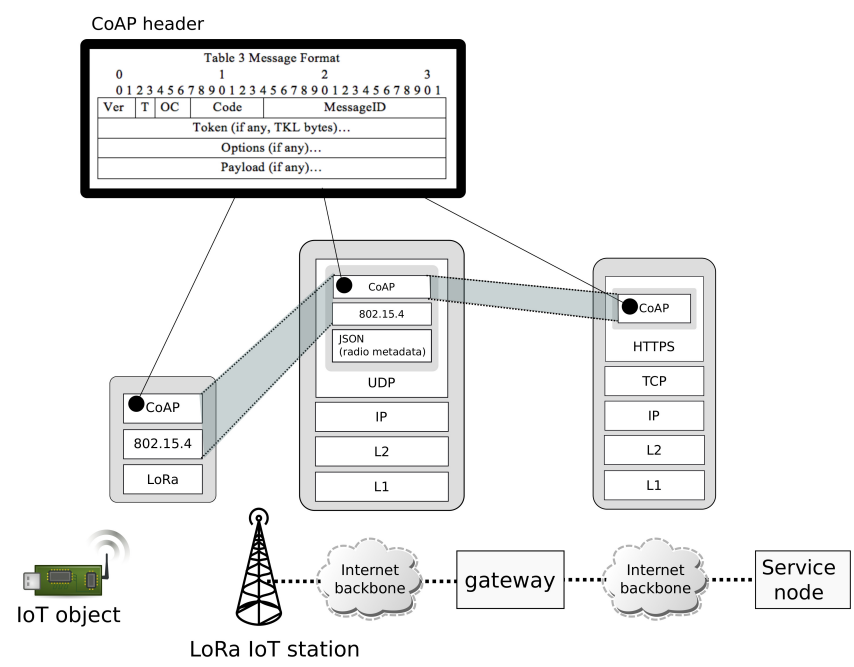

Fig. 2. LoRa FABIAN protocol stacks

\section{Testing of the LoRA FABIAN network}

These measurements are supposed to give us insight about the Quality of Service (QOS) that the LoRa FABIAN (using LoRA ${ }^{\mathrm{TM}}$ technology) network can provide, and give us some statistical data for different configurations of the network. Other than that, they can also bring to light some of the factors that need to be considered when testing these kinds of networks and provide some statistical data for different configurations of the network.

For this, a number of measurements were performed in order to get basic metrics that can be used to describe the QoS, such as the Packet Error Rate (PER), but also some parameters related to the Radio and LoRa layers, like the Signal to Noise Ratio (SNR), and the Received Signal Strength Indicator (RSSI).

At the time of performing the tests, all the communication within the LoRa FABIAN network occurred on

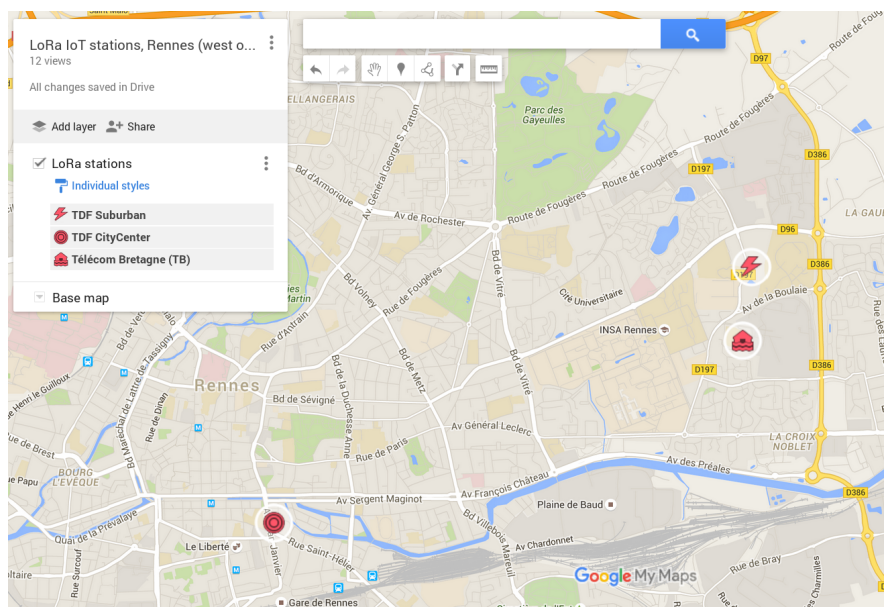

Fig. 3. LoRa IoT stations in LoRa FABIAN network in area of Rennes city, northwest of France

the $868.1 \mathrm{KHz}$ frequency band. The bandwidth that was used was $125 \mathrm{kHz}$. These parameters, as well as the coding rate (that was set to $4 / 5)$, and the packet payload (25 bytes, including MAC frame) remained fixed throughout the measurements. The parameters that were varied are: spreading factor (SF), distance, surroundings, antenna size on IoT object and elevation/location of LoRa IoT stations (by observing results on differently placed stations).

With this simple setup, we were able to generate traffic really similar to one that can be used by real application (like sensor monitoring), and then, from that, extract QoS metrics within various conditions. For this round of measurements we chose to focus on testing only the uplink (from IoT object to the LoRa IoT station), as this is the more common case in IoT.

First we did a basic test to get a rough idea about the range and performance of the three LoRa IoT stations (TDF Suburban, TDF CityCenter and TB on Figure 3), based on the station location but also on the distance, SF, and the type of antenna used on the IoT object. Following that, we gathered some statistical data of performance of LoRa for a certain distance (but with varying the location and surroundings). This data allowed us to evaluate the different parameters (such as SNR and RSSI) in these scenarios, but also provided us with insight into various influences on the performance.

\section{A. Range tests}

To perform these measurements we moved on a trajectory (going up to roughly $3 \mathrm{~km}$ away from TDF Suburban and TB stations, and $6 \mathrm{~km}$ away from the TDF CityCenter one, shown by pins on Fig. 4 and 5), with two IoT nodes that were periodically sending packets. One node (that we call Gamma) had a soldered-on antenna, while the other one (that we call Beta) had a weaker, on-board, antenna. For the first round Gamma was using SF 12 while Beta was using SF 10. In order to isolate the influence of the different 


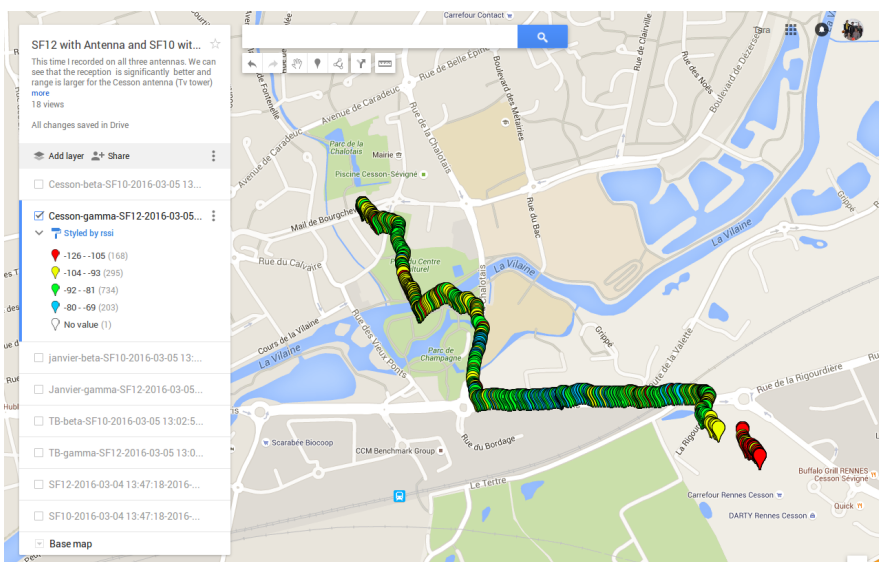

Fig. 4. Received packets on TDF Suburban LoRa station

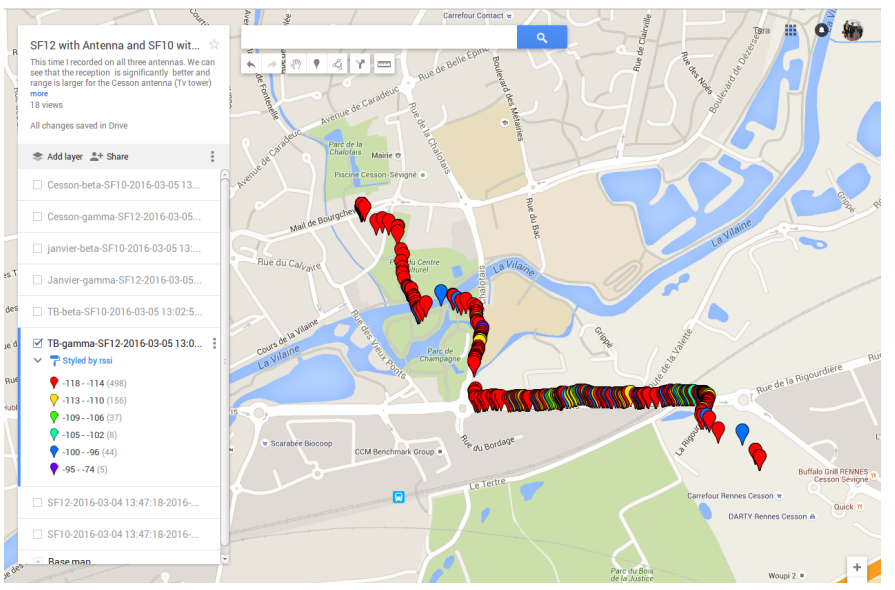

Fig. 5. Received packets on Télécom Bretagne (TB) LoRa station

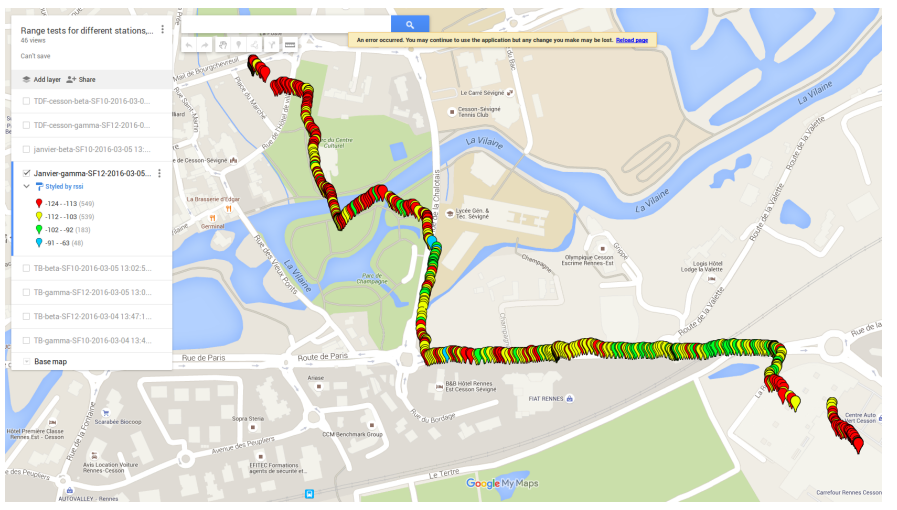

Fig. 6. Received packets on LoRa station in the center of Rennes (TDF CityCenter)

node antenna, the measurement was repeated, but with switching the SF's (Gamma used SF 10 and Beta SF 12)

All three LoRa stations (positioned as shown in Figure 3) were listening, and logging the information (detailed in Appendix A most importantly - RSSI and SNR) for

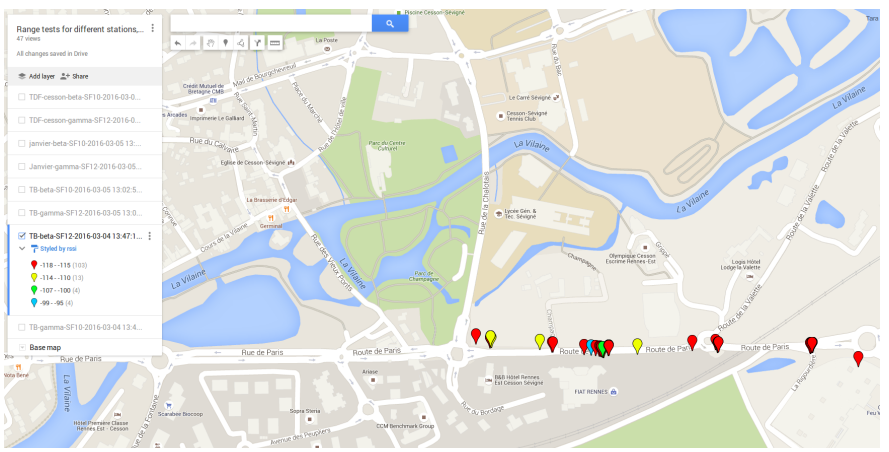

Fig. 7. Received packets on Télécom Bretagne LoRa station with on-board antenna

each received packet. In this way we could compare the reception on each LoRa station. Since two stations (TB and TDF Suburban) were relatively close to each other, we could observe the influence of their placement (most notably, the elevationa).

The results from the three stations, when receiving from Gamma (configured with SF 12), can be seen in Figures 4. 5 and 6. Each pin represents one packet, as received on the given station. The pin color represents the RSSI of the packet. Red is used for packets received between -120 and -106 dBm's, yellow for the range -105 to $-94 \mathrm{dBm}$ 's and green for the range -93 to $-82 \mathrm{dBm}$ 's and finally blue for the range between -81 to $-69 \mathrm{dBm}$ 's. (To access all the data, interactively on the map or to download it, go to [18]).

By comparing the results on the TB station (elevation $55 \mathrm{~m}$ ) and on the TDF Suburban station (elevation 160m), we can see that the elevation of the antenna makes a dramatic difference. At some areas along the road, there is complete blackout for the TB station (mainly in valleys), while TDF Suburban station's reception remains active throughout the whole trajectory. During the entire measurement, in total, 1447 packets were sent. Out of that, TB station received only 748 , while the TDF Suburban station received 1400. Maybe surprisingly, the station in the city center of Rennes (TDF CityCenter, elevation 85m) that is around $6 \mathrm{~km}$ away from the farthest point, received 1319 packets. We can also observe that the RSSI given by the TB station, throughout the whole route, rarely goes over $-112 \mathrm{dBm}$, while on the TDF Suburban, more than half of the packets are received with RSSI between -92 and -81 $\mathrm{dBm}$. It is interesting to note that the TDF CityCenter station is able to receive packets with RSSI below -124 $\mathrm{dBm}$. Moreover, most of the packets (1088 out of 1319), on this station, are received in range between -124 and $103 \mathrm{dBm}$. This shows us that, in some cases, even thought the average RSSI is worse, the reception can be better.

Figure 7 shows the result on the TB station, for the same SF, when using Beta (on-board antenna), rather than Gamma. In this case only 59 out of 1000 packets were received, showing that the use of a soldered-on antenna makes a significant difference, with the success rate jump- 


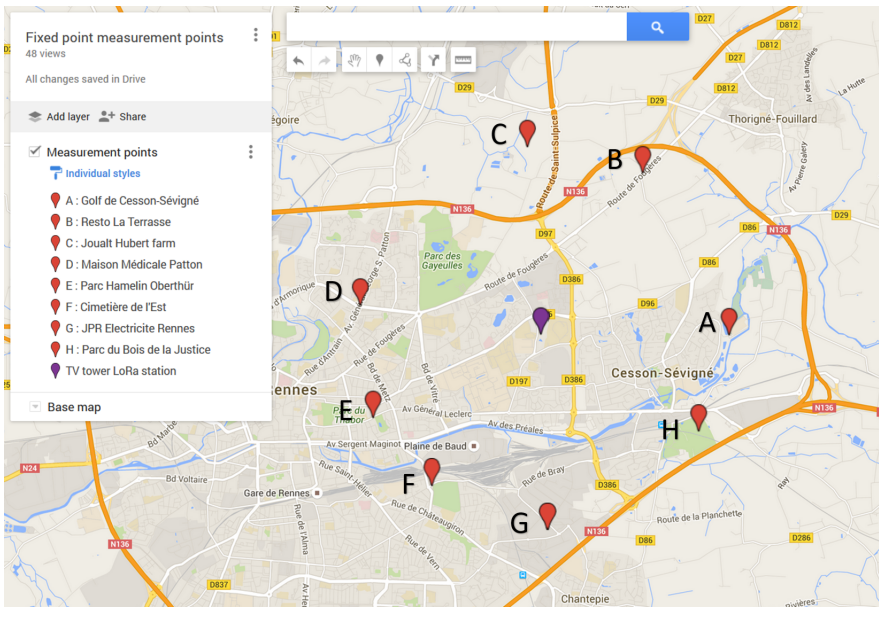

Fig. 8. Fixed measurment points, $3 \mathrm{~km}$ distance from TV tower LoRa IoT station

ing from $6 \%$ to $62 \%$ when adding the antenna.

\section{B. Fixed point measurements}

In the fixed point measurement we sought to take a closer look at the influence of location and environment on the performance of the LoRa network, so we chose 8 points (as shown on map in Fig 8 ) that were on the same distance $(3 \mathrm{~km})$ from the LoRa station we chose to focus on - TDF Suburban.

Half of these points are located in a more urban part of Rennes, while the other half is on the borders or outside. The environment varied from suburb-like found in Cesson-Sevigne, to ones mostly empty (with but roadside restaurants), to a farm in a rural environment, and finally to points in the city of Rennes.

On each of these measuring points, 500 packets (each having a 25 byte payload) were sent out for SF 7,9 and 10. Gamma (with soldered-on antenna) was using SF 10 to send 500 packets, meanwhile Beta (with on-board antenna) was sending 500 packets for both SF7 and SF9.

This allowed us, to gather some statistical data about the performance of the LoRa network on distance of $3 \mathrm{~km}$ in different areas. The results are synthesized in Table $\mathrm{I}$.

It was expected that in urban areas the results are going to be worse, and this was proven true by the packet error rate (PER) not going under $40 \%$ in this areas, however what is a bit surprising is the dramatic degradation of results in non-urban (even can be called rural) areas, such as the Joualt Hubert farm (C on Fig. 8) or Parc du Bois de la Justice ( $\mathrm{H}$ on Fig. 8), with the PER worsening by $30 \%$ as compared to Golf de Cesson (A on Fig. 8). This might be partly explained by the elevation profile between the LoRa station and measurement point. Namely, when the elevation profile between the measurement point and the LoRa station is strictly degrading - the reception is good. When some "hills" are present that might block the reception - it is much worse. In B you can find elevation

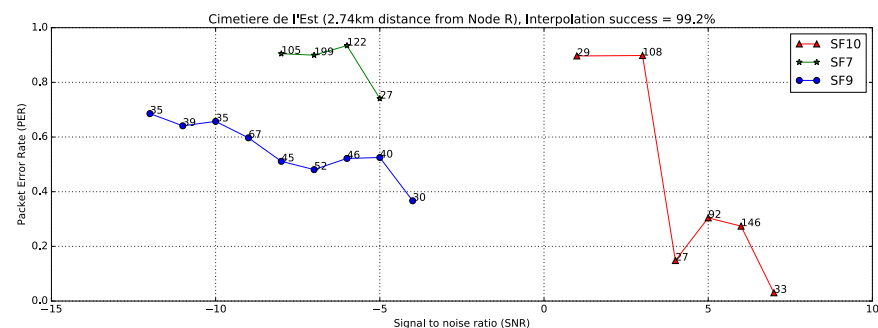

Fig. 9. Correlation between PER and SNR, $3 \mathrm{~km}$ from the LoRa IoT station

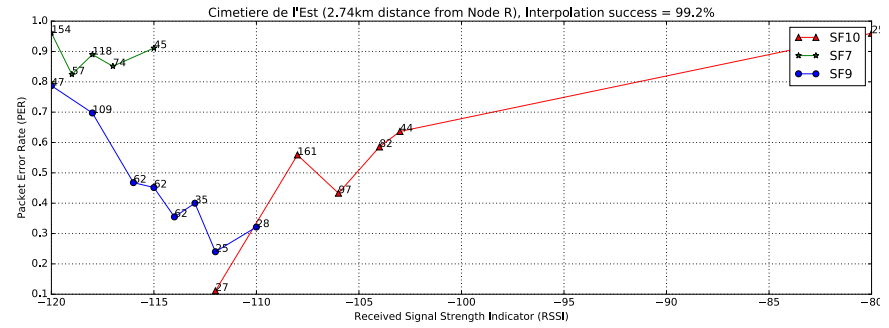

Fig. 10. Correlation between PER and RSSI, 3km from the LoRa IoT station

profiles between non-urban measurement points and the TDF Suburban station.

It can also be noted that the correlation between RSSI and PER is not straightforward. Namely, sometimes even when the RSSI mean is high the PER is going to be worse. This is more obvious by looking at a graph such as in Figure 10 showing the overall PER for each RSSI value at a certain location (the annotations on each point denote the number of packets received - both successfully and unsuccessfully - for a given RSSI) On the other hand, SNR (Fig. 9), since it takes into account also the current noise level, shows the expected property - more packets are lost when the SNR is lower.

All the scripts used for parsing, plotting and analyzing data can be found on 19 .

\section{Conclusion and Future work}

In this paper, we describe our experimental LoRa setup in the city of Rennes - LoRa FABIAN, and we design, perform and analyse measurements for it. We propose a plot of the PER as a function of the SNR, for some system conditions. There are many measurements that can still be done to further improve characterization of LoRa networks. Beside trying to find the correlation between elevation and performance, many other factors, such as influence of small-scale fading should be validated before proposing a channel model for LoRa and more generally for LPWANs. Measurement for other LPWAN devices (using other physical layer technology) also should be done, and are planned for our team. Some parameters have still to be varied and analyzed, for example : packet size, bandwidth and coding rate. 
TABLE I. Synthesis of MEASUREMENTS

\begin{tabular}{|c|c|c|c|c|c|c|c|}
\hline Point & SF & $\operatorname{RSSI}(\mathrm{dBm})$ mean & RSSI std. dev & $\mathrm{SNR}(\mathrm{dB})$ mean & SNR std. dev. & PER & Packets $\mathrm{Rx} / \mathrm{Tx}$ \\
\hline \multirow{3}{*}{ Cemeterie Est } & SF10 & -106.52 & 4.74 & 5.17 & 2.92 & $44.0 \%$ & $280 / 500$ \\
\hline & SF7 & -117.81 & 1.42 & -7.08 & 1.12 & $90.4 \%$ & $48 / 500$ \\
\hline & SF9 & -113.68 & 3.71 & -6.08 & 4.46 & $51.0 \%$ & $245 / 500$ \\
\hline \multirow{2}{*}{ La Terasse } & SF10 & -100.51 & 3.64 & 6.47 & 1.54 & $3.2 \%$ & $484 / 500$ \\
\hline & SF7 & -107.75 & 3.93 & 2.86 & 3.85 & $47.0 \%$ & $265 / 500$ \\
\hline \multirow{3}{*}{$\begin{array}{l}\text { Rue de la } \\
\text { Frebardiere }\end{array}$} & SF10 & -106.20 & 5.89 & 5.10 & 3.03 & $53.4 \%$ & $233 / 500$ \\
\hline & SF7 & -111.63 & 3.33 & -0.00 & 3.90 & $33.8 \%$ & $331 / 500$ \\
\hline & SF9 & -109.74 & 3.55 & -0.72 & 4.75 & $35.2 \%$ & $324 / 500$ \\
\hline \multirow{3}{*}{ Maison Medicale } & SF10 & -109.87 & 4.15 & 3.71 & 2.66 & $44.2 \%$ & $279 / 500$ \\
\hline & SF7 & & & & & $100.0 \%$ & $0 / 500$ \\
\hline & SF9 & -116.06 & 2.82 & -9.33 & 2.97 & $87.2 \%$ & $64 / 500$ \\
\hline \multirow{3}{*}{ Parc Hamelin } & SF10 & -109.45 & 4.68 & 4.02 & 3.13 & $41.6 \%$ & $292 / 500$ \\
\hline & SF7 & -115.70 & 2.48 & -4.93 & 1.94 & $57.2 \%$ & $214 / 500$ \\
\hline & SF9 & -113.51 & 3.74 & -4.85 & 3.80 & $43.0 \%$ & $285 / 500$ \\
\hline \multirow{3}{*}{$\begin{array}{l}\text { Parc du Bois } \\
\text { de la Justice }\end{array}$} & SF10 & -107.67 & 4.33 & 5.03 & 2.38 & $33.6 \%$ & $332 / 500$ \\
\hline & SF7 & -113.97 & 2.80 & -2.13 & 3.10 & $49.2 \%$ & $254 / 500$ \\
\hline & SF9 & -110.85 & 4.72 & -3.40 & 4.56 & $21.8 \%$ & $391 / 500$ \\
\hline Golf de Cesson & SF 10 & -106.25 & 4.82 & 5.46 & 2.58 & $3.0 \%$ & $485 / 500$ \\
\hline \multirow{3}{*}{ Joualt Hubert farm } & SF10 & -106.82 & 5.55 & 4.73 & 2.93 & $35.4 \%$ & $323 / 500$ \\
\hline & SF7 & -114.01 & 2.73 & -2.51 & 2.78 & $42.2 \%$ & $289 / 500$ \\
\hline & SF9 & -114.30 & 3.65 & -6.08 & 3.58 & $49.2 \%$ & $254 / 500$ \\
\hline
\end{tabular}

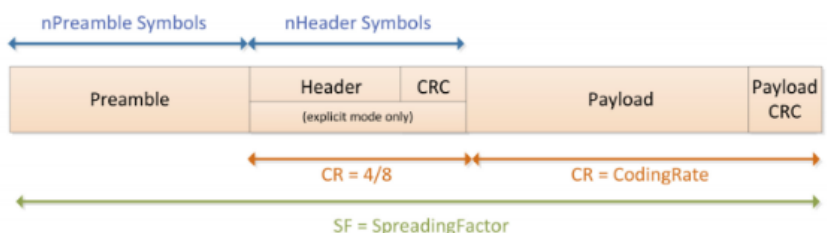

Fig. 11. LoRa packet structure

Another important open issue for LoRa networks is that, to best of our knowledge, there is no established LoRa station selection method. In the current setup of our network, when transmitting to the IoT object (downlink), all three LoRa stations are used to transmit the same packet. This is inefficient and undesired, especially when using freely-available unlicensed bands. However, it is still unclear by which parameters the selection should be done. We have shown, for example, that higher RSSI doesn't necessarily lead to better PER. Devising a LoRa station selection algorithm would be an interesting next step.

\section{APPENDix A \\ LORA PACKET STRUCTURE}

Figure 11 shows the format of the packet in the LoRa network. The first field is the preamble, which is in charge of the synchronization of the receiver and the incoming packet. The next field is the header which provides information such as the SF used, the FEC code rate and size of the payload. Then, the payload field containing the data to be transmitted and finally the last field is a cyclic redundancy check (CRC) for error detection.

The structure may vary depending on whether explicit header is used, and also on the length of the preamble (currently, we are using 8 symbols)

\section{A. Example of packet received by LoRa IoT station}

Packets flowing into the LoRa FABIAN network are received and sent by LoRa stations. In this section, we

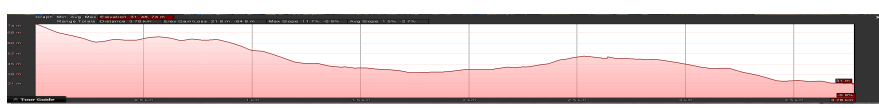

Fig. 12. Elevation Profile between TDF Suburban LoRa station (left) and point A (see Fig. 8

will present some example of LoRa FABIAN data, as they are received by one LoRa station. We will start by a simple raw example and then describe the LoRa layer meta data, and the 802.15.4 and CoAP data.

NEW PACKET: received packet (size 43, modulation 16, BW 2, DR 2, RSSI -89.0)

JSON up: \{"rxpk": [\{"tmst":3936040845,

"time" : "2016-01-25T16:40:15.164887Z", "chan" : 8,

"rfch" : 0, "freq" : 868.100000, "stat" : 1,

"modu" : "LORA", "datr" : "SF7BW250", "codr" : "4/7",

"Isnr" : 8.2, "rssi":-89, "size" :43,

"data" : "IjYB+rAAAAAAAAgAAEYBqv9MxGidNU49AX

RoZXRhLnQuZXUub3Jng25vMg==" \}] \}

Message in HEX. Size: 43

223601FAB000000000000800004601AAFF4CC4689D3 54E3D0174686574612E742E65752E6F7267836E6F32

In this example, we are able to see the meta data in JSON format, as made available by the LoRa Station and the Semtech engine 20]. Description of each field can be found on 20

We can also see the payload of the LoRa data, including thus the IEEE 802.15.4 [21] layer and the CoAP one. Table III shows the interpretation.

\section{Appendix B}

\section{ELEVATION PROFILES}

Figures 12 show the elevation profiles between the TDF Suburban LoRa station and the non-urban measurement points as calculated by Google Earth 22 software. 
TABLE II. 802.15.4 FRAME WITH COAP PAYLOAD

\begin{tabular}{ll} 
Message in HEX & Interpretation \\
\hline 223601 & $\begin{array}{l}\text { IEEE 802.15.4 Header: Frame control } \\
\text { field and sequence number }\end{array}$ \\
\hline FAB0000000000008 & $\begin{array}{l}\text { IEEE 802.15.4 Destination address (8 } \\
\text { Bytes addressing mode) }\end{array}$ \\
\hline 0000 & $\begin{array}{l}\text { IEEE 802.15.4 Source Address (2 Bytes } \\
\text { addressing mode) }\end{array}$ \\
\hline $\begin{array}{l}\text { 04601AAFF4CC46 } \\
\text { 89D354E3D01746 } \\
\text { 86574612E742E65 } \\
\text { F52E67267836E6 }\end{array}$ & \\
\hline
\end{tabular}

Fig. 13. Elevation Profile between TDF Suburban LoRa station (left) and point B (see Fig. 8)

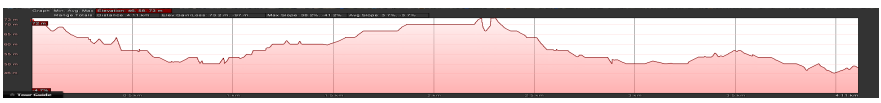

Fig. 14. Elevation Profile between TDF Suburban LoRa station (left) and point C (see Fig. 8

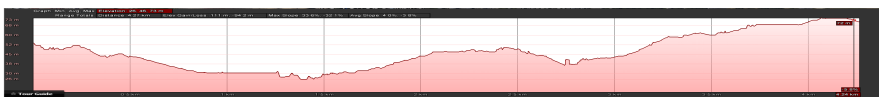

Fig. 15. Elevation Profile between TDF Suburban LoRa station (left) and point $\mathrm{H}$ (see Fig. 8)

\section{REFERENCES}

[1] Lora Alliance web page. [Online]. Available: https://www.lora-alliance.org/ (visited on Apr. 5, 2016).

[2] L. Vangelista, A. Zanella, and M. Zorzi, "Long-range IoT technologies: The dawn of LoRa ${ }^{\mathrm{TM}}$," in Future Access Enablers for Ubiquitous and Intelligent Infrastructures, Springer, 2015, pp. 51-58.

[3] M. Centenaro, L. Vangelista, A. Zanella, and M. Zorzi, "Long-range communications in unlicensed bands: The rising stars in the IoT and smart city scenarios," ArXiv preprint arXiv:1510.00620, 2015.

[4] T. Wendt, F. Volk, and E. Mackensen, "A benchmark survey of long range (LoRa ${ }^{\mathrm{TM}}$ ) spread-spectrum communication at $2.45 \mathrm{ghz}$ for safety applications," in Wireless and Microwave Technology Conference (WAMICON), 2015 IEEE 16th Annual, IEEE, 2015, pp. 1-4.

[5] M. Aref and A. Sikora, "Free space range measurements with Semtech LoRa ${ }^{\mathrm{TM}}$ technology," in Wireless Systems within the Conferences on Intelligent Data Acquisition and Advanced Computing Systems: Technology and Applications (IDAACS-SWS), 2014 2nd International Symposium on, IEEE, 2014, pp. 19-23.

[6] LoRa IoT Station $868 \mathrm{MHz}$ - Kerlink / M2m : Solutions de communication machinetomachine. [Online]. Available: http://www.kerlink.fr/fr/produits/lora-iotstation/lora-iot-station-868-mhz (visited on Mar. 16, 2016).
[7] (). Wi6labs, [Online]. Available: http://www.wi6labs.com/ (visited on May 6, 2016).

[8] Réseau Fabian - L'Internet des Objets dans la ville. [Online]. Available: http://www.reseaufabian.fr/ (visited on Mar. 15, 2016).

[9] Arduino - ArduinoBoardUno. [Online]. Available: https://www.arduino.cc/en/Main/ArduinoBoardUno (visited on Mar. 16, 2016).

[10] Froggy Factory. [Online]. Available: http://www.froggyfactory.com/froggy/ (visited on Mar. 16, 2016).

[11] Wiblabs/lorafabian code repository. [Online]. Available: https://github.com/Wi6labs/lorafabian (visited on Mar. 16, 2016).

[12] A. Pelov, L. Toutain, and Y. Delibie, Constrained Signaling Over LP-WAN, Internet Engineering Task Force. [Online]. Available:

https://tools.ietf.org/html/draft-pelov-core-cosol-01 (visited on Mar. 16, 2016).

[13] Z. Shelby, K. Hartke, and C. Bormann, The constrained application protocol (CoAP), RFC 7252 (Proposed Standard), Internet Engineering Task Force, Jun. 2014. [Online]. Available: http://www.ietf.org/rfc/rfc7252.txt

[14] REpresentational State Transfer, en, Page Version ID: 709559774, Mar. 2016. [Online]. Available:

https://en.wikipedia.org/w/index.php?title= Representational_state_transfer\&oldid $=709559774$ (visited on Mar. 16, 2016).

[15] JavaScript Object Notation - Wikipedia, the free encyclopedia. [Online]. Available:

https://en.wikipedia.org/wiki/JSON (visited on Apr. 10, 2016).

[16] Californium (Cf) CoAP framework. [Online]. Available: https://www.eclipse.org/californium/ (visited on Mar. 16, 2016).

[17] Télédiffusion de france. [Online]. Available: http://www.tdf.fr/ (visited on Mar. 16, 2016).

[18] Range tests for different stations, node antennas and $S F$ 's, interactive map. [Online]. Available: https://drive.google.com/open?id=1_30lffhl8i49GLAaq-KUkrWtE8\&usp=-sharing (visited on Mar. 8, 2016).

[19] T. Petric, Code repository of LoRa station output parser, https://github.com/yotf/NodeR-parse, 2016.

[20] Description of JSON metadata on Semtech's LoRa-net code repository, https://github.com/Loranet/packet_forwarder/blob/master/PROTOCOL.TXT (visited on Mar. 15, 2016).

[21] IEEE 802.15.4, en, Page Version ID: 704926777, Feb. 2016. [Online]. Available: https://en.wikipedia.org/w/ index.php?title=IEEE_802.15.4\&oldid $=704926777$ (visited on Mar. 16, 2016).

[22] Google Earth. [Online]. Available: https://www.google.fr/intl/fr/earth/ (visited on Apr. 27, 2016). 